\title{
A Thin-film Magnetoresistive Angle Detector
}

\author{
KEES J M EIJKEL, JOHAN W WTEBERDINK, JAN H J FLUITMAN and THEO J A POPMA \\ Unversity of Twente EL-TDM, P O Box 217, 7500 AE Enschede (The Netherlands)
}

PETER GROOT and HENK LEEUWIS

Twente Technology Transfer (3T) BV, PO Box 545, 7500 AM Enschede (The Netherlands)

\begin{abstract}
An overview is given of the results of our research on a contactless angle detector based on the anisotropic magnetoresistance effect (AMR effect) in a permalloy thin film The results of high-temperature annealing treatment of the permalloy film are discussed Such a treatment suppresses the effects of the uniaxial magnetic anisotropy that is present in a permalloy thin film and increases the AMR effect, thus improving the detector signal The performance of the detector throughout a temperature range of 20 to $120^{\circ} \mathrm{C}$ and the results of heat treatment at $125^{\circ} \mathrm{C}$ for 1 week have been tested
\end{abstract}

\section{Introduction}

Over the past 5 years, a contactless angle detector based on the anisotropic magnetoresistance effect (AMR effect) in a permalloy thin film has been proposed and developed $[1,2]$ This paper presents an overview of the results of our research on this detector and discloses recent unpublished results

The angle detector consists of a pair of ident1cal pseudo-Hall devices (PHDs), or equivalent devices such as magnetoresistor bridges, mutually rotated through $45^{\circ}$ and positioned opposite a rotatable permanent magnet The magnetic field at the position of the PHDs is largely in-plane

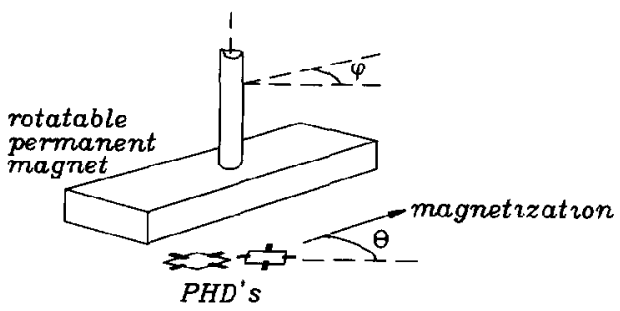

Fig 1 The angle detector, consisting of a pair of pseudo-Hall devices opposite a rotable permanent magnet
(Fig 1) The magnetization angle $\Theta$ in the PHDs reflects the angular position $\varphi$ of the rotatable magnet and is comprised in the output signals of the PHDs

$\left.\begin{array}{l}V_{1}=I \Delta \rho k \sin (2 \Theta) \\ V_{2}=I \Delta \rho k \cos (2 \Theta)\end{array}\right\}$

in which $I$ is the driving current of the PHDs and $k$ is a geometrical factor The output angle of the detector, $\Theta$, is independent of temperature, in principle The magnetoresistivity $\Delta \rho$ and resistivity $\rho$ of the permalloy are defined as

$\Delta \rho=\left(\rho_{\|}-\rho_{\perp}\right) / 2$ and $\rho=\left(\rho_{\|}+\rho_{\perp} / 2\right)$

in which $\rho_{\|}$and $\rho_{\perp}$ are the resistivity parallel and perpendicular to the magnetization in the permalloy The permalloy thin films are sputtered in the magnetostriction-free composition ( 81 at $\% \mathrm{~N}_{1}$ and 19 at $\% \mathrm{Fe}$ ) This ensures a low influence of temperature-induced or mechanically-induced stresses in the film The values of $\Delta \rho$ and $\rho$ are $32 \times 10^{-9} \Omega \mathrm{m}$ and $22 \times 10^{-7} \Omega \mathrm{m}$ in a fresh film

The performance of an angle detector as described above depends on its magnetical and electrical properties The magnetical properties of the detector include the strength and homogeneity of the magnetic field of the magnet, on the one hand, and the magnetic properties of the permalloy thin film on the other The electrical properties of the detector, which have been discussed elsewhere [3], are determined by the magnetoresistıve properties of the permalloy and the geometry of the PHDs Of course, the stability of all these properties is of major importance for the performance of the detector The electrical and magnetical properties of the system can be treated separately, because their mutual interaction (normal Hall effect, magnetic fields caused by the currents in the thin film etc) is negligible

\section{Magnetic Behavior}

A few short remarks concerning the permanent magnet should be made The magnetic field at the

(C) Elsevier Sequoia/Printed in The Netherlands 
position of the PHDs should be large to ensure a small influence of the earth's magnetic field and other disturbing a $c$ or d c fields, the field should be stable (no demagnetization) and it should be homogeneous to enable a homogeneous magnetization of the PHDs These requirements can be met using common magnetic materials like anisotropic ferroxdure In practical situations, errors caused by disturbing magnetic fields (e $\mathrm{g}$ the earth's magnetic field) or by the inhomogeneity of the field can be kept below $01^{\circ}$ [1] If very large field strengths and high stability of the magnet are required, new magnetic materials like $\mathrm{SmCo}_{5}$ and $\mathrm{NdFeB}$ can be applied

Permalloy films show magnetic anisotropy, which causes an onentation difference between the magnetization and the magnetic field, resulting in an angle detector measurement error The magnetic anisotropy can be onented if the film is deposited in the presence of a magnetic field [4] Under normal deposition conditions, the anisotropy field strength $H_{\mathrm{k}}$ in a sputtered or evaporated film is about $350 \mathrm{~A} / \mathrm{m}$, leading to a maxımum angle detector measurement error of about $05^{\circ}$ if a magnetic field of $20 \mathrm{kA} / \mathrm{m}$ is used In many cases this error is not acceptable $\mathbf{A}$ number of technques have been developed to reduce this error [1] The use of a permalloy bilayer with perpendicular in-plane onentation of the anisotropy in both sublayers proved to be very successful The results have been published elsewhere [5]

Alternatively, one can use a permalloy film consisting of many small areas with randomly oriented magnetic anisotropy If such a film experiences a sufficiently large in-plane magnetic field (larger than the local anisotropy field $H_{k}$ ) with arbitrary orientation, the mean magnetization in the film will have the orientation of the field [1] To manufacture such a magnetically isotropic film, deposition of the permalloy in a rotating magnetic field was considered [6] This technique was not successful We discovered in several experiments that a permalloy layer with a welldefined anisotropy dictates the anisotropy orientation of a second layer sputtered on top of the first, even in a moderate $(2 \mathrm{kA} / \mathrm{m})$ in-plane magnetic field of arbitrary but constant orientation This indicates that the orientation of the anisotropy is transferred to the second layer by means of some nonmagnetic interaction (e $\mathrm{g}$ by the surface structure of the first film) or that the magnetic anisotropy is confined to and transferred by certain (small) areas in the film which are not influenced at the field strength used The fact that Goto et al [7] managed to reonent the anisotropy in the second layer using a stronger deposition field indicates that the latter is valid or that a balance exists between field-induced and structure-induced anisotropy

Another method to produce a magnetically 1sotropic permalloy film is the use of a hightemperature annealing step to disonent the anisotropy locally inside the permalloy layer Experiments performed by Metzdorf [8] show that changes in anisotropy orientation, induced by annealing in a magnetic field at temperatures up to $400^{\circ} \mathrm{C}$, are partly reversible when annealing at lower temperatures Stable films are obtained after annealıng at temperatures above $400^{\circ} \mathrm{C}$ [4] In our case, a desorientation of the magnetization in the permalloy is necessary during annealing This can be achieved by exceeding the Cune temperature (for permalloy at a 81/19 composition, just above $500^{\circ} \mathrm{C}$ ) We annealed a number of films at different temperatures between $200^{\circ} \mathrm{C}$ to $600^{\circ} \mathrm{C}$ for $1 \mathrm{~h}$ and measured the coercivity $H_{\mathrm{c}}$ of the film (using an inductive hysteresis loop tracer), $\alpha_{90}$ (the angle which includes $90 \%$ of all anisotropy onentations in the film, measured using the Crowther method [9]), and the maximum angle between the magnetic field and the mean magnetization in the $(\varphi-\Theta)_{\max }$, at a field strength of $2 \mathrm{kA} / \mathrm{m}$ using
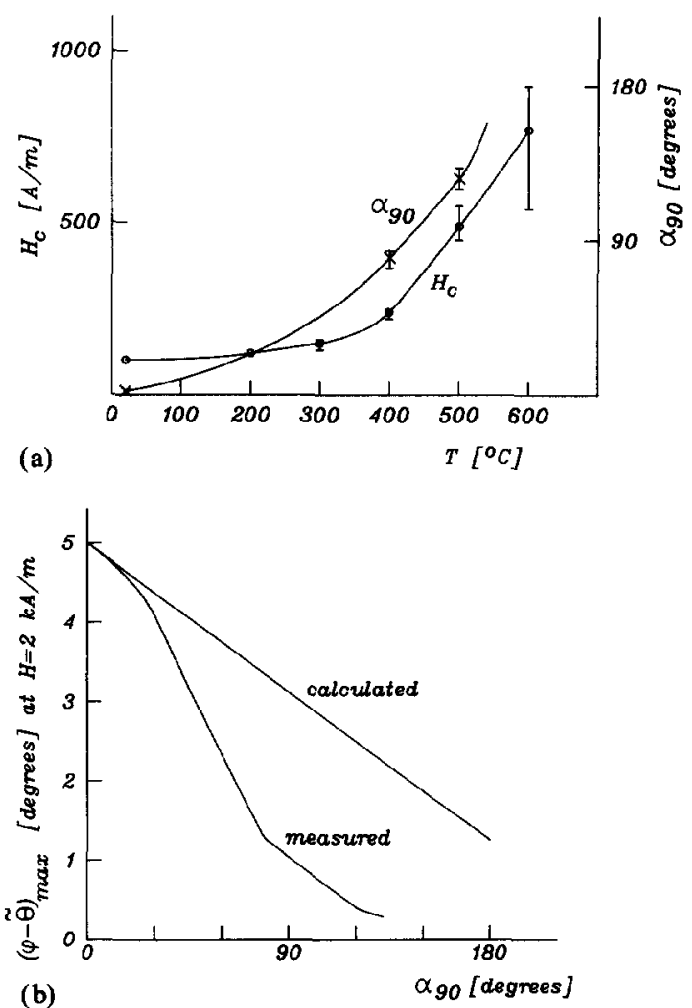

Fig 2 (a) $H_{\mathrm{c}}$ and $\alpha_{90}$ as a function of annealing temperature (annealing time $1 \mathrm{~h}$ ) The error bars indicate the spread of the results (b) Measured and calculated values of $(\varphi-\theta)_{\max }$ as a function of $\alpha_{90}$ in a field of $2 \mathrm{kA} / \mathrm{m}$ 
the measurement system described in ref 10 The films were annealed in a nitrogen atmosphere and were protected by a 75-nm SiO film The results are shown in Fig 2(a) and (b) It is clear that $\alpha_{90}$ increases with increasing annealing temperature, as expected The films annealed at $600^{\circ} \mathrm{C}$ are magnetically isotropic In these films, $\alpha_{90}$ cannot be determined with the Crowther method

To gain more insight into the properties of the annealed films, we performed computer simulations of films consisting of a large number of small areas with no mutual magnetic interaction, each having a well-defined magnetic anisotropy $\left(H_{k}\right.$ equal to that of a fresh film, $350 \mathrm{~A} / \mathrm{m}$ ), with an orientation distribution as measured in our annealed films The calculated values of $(\varphi-\Theta)_{\max }$ at a field strength of $2 \mathrm{kA} / \mathrm{m}$ are shown in $F_{1 g}$ 2(b) Two possible mechanisms can cause the observed differences

-The anisotropy field strength of small areas with well-defined anisotropy decreases during annealing

-A large fraction of the total amount of these areas has random anisotropy onentation This fraction is not visible in measurements of $(\varphi-\tilde{\Theta})_{\max }$ or in the Crowther measurements, but it reduces the influence of the remaining part of the film on the orientation of the mean magnetization

The areas with well-defined anisotropy onentation in high-temperature $\left(\geqslant 500^{\circ} \mathrm{C}\right)$ annealed films are small In Kerr rotation measurements using a small light spot of a few tens of micrometers, comparable magnetic behavior in different areas is found

Deterioration of the permalloy in high-temperature annealed films could be responsible for the observed increase in coercivity Capacitor structures of $100 \times 100 \mu \mathrm{m}$ formed by a permalloy film, a 75-nm SiO layer and an aluminum layer showed low resistance in some cases, but good Isolation in some other cases, all on the same wafer This indicates that the $\mathrm{S}, \mathrm{O}$ layer is not completely closed (e g due to pin-holes) No visible damage to the film surface could be found with microscopic inspection and SEM

It is concluded that a film annealed at $500^{\circ} \mathrm{C}$ for $1 \mathrm{~h}$ is appropriate for use in an angle detector $\left((\varphi-\Theta)_{\max }<01^{\circ}\right.$ for $\left.H=20 \mathrm{kA} / \mathrm{m}\right) \quad \mathrm{High}-$ temperature annealing has another advantage it lowers the resistivity of the film [11], thus increasing the magnetoresistivity ratio $\Delta \rho / \rho$ from $15 \%$ in fresh films to $2 \%$

\section{Technology}

The manufacture of our PHDs and magnetoresistor bridges is straightforward A permalloy film is deposited by RF sputtering on an oxidized silicon wafer The film geometry is produced using photolithographic tochniques and wet chemical etching Film and substrate are covered with a $75-\mathrm{nm}$ SiO film The wafer is annealed for $2 \mathrm{~h}$ at $500^{\circ} \mathrm{C}$ and a stable, magnetically isotropic film is obtained $\mathrm{V} 1 \mathrm{a}$-holes are etched in the $\mathrm{SiO}$ layer by reactive-ion etching in a $\mathrm{CF}_{4} / \mathrm{O}_{2}$ atmosphere The permalloy is backsputtered to provide a clean surface for the contacts Stable contacts with low interdiffusion at high temperatures can be produced using Mo and $\mathrm{Cr}$ as a contact matenal [12] We use a 25-nm chromium film followed by a $05-\mu \mathrm{m}$ aluminum layer The aluminum and chromium are wet-etched using the same photoresist mark The wafer is sliced and the devices are mounted on a substrate

\section{Stability}

The stability of the electrical and magnetic properties of the devices can be determined in two ways In order to determine the behavior of the devices at different temperatures from $20^{\circ} \mathrm{C}$ to $120^{\circ} \mathrm{C}$, the devices were mounted on a heating plate in an angle detector set-up [10] Alternatively, the devices were heated to $125^{\circ} \mathrm{C}$ and their properties measured after 1,2 and 7 days All measurements were performed on PHDs of $3 \times 3$ and $1 \times 1 \mathrm{~mm}$

Concerning the magnetic anisotropy in the films, it could be concluded that small anisotropy could be induced by annealing at $125^{\circ} \mathrm{C}$ in a magnetic field The resulting values of $(\varphi-\widetilde{\Theta})_{\max }$ in different films in a field of $20 \mathrm{kA} / \mathrm{m}$ were determined to be well below $01^{\circ}$ for all films

Devices with a large overlap of the contact material over the SiO-covered permalloy showed large, irreversible changes in signal amplitude and offset dunng heat treatment, presumably due to the fact that the $\mathrm{S}_{1} \mathrm{O}$ layer was not completely closed The following results concern devices with a small overlap which showed good stability

Amplitude imbalance between two as-manufactured devices in an angle detector is usually of the order of $1-5 \%$ Temperature-induced imbalance in the temperature range of 20 to $120^{\circ} \mathrm{C}$ shows a maximum of $05 \%$, yielding a measurement error of less than $01^{\circ}$

Offset stability in the devices is very critical, because of the sensitivity of the offset voltage to geometrical vanations The offset was trimmed to zero at $20^{\circ} \mathrm{C}$ using the correction circuit shown in $F_{1 g} 3$ In subsequent measurements, the trimmed devices showed an offset of less than $1 \%$ in the temperature range $20^{\circ} \mathrm{C}$ to $120^{\circ} \mathrm{C}$ The offset changes are reversible and are attributed to position-dependent changes in the properties of 


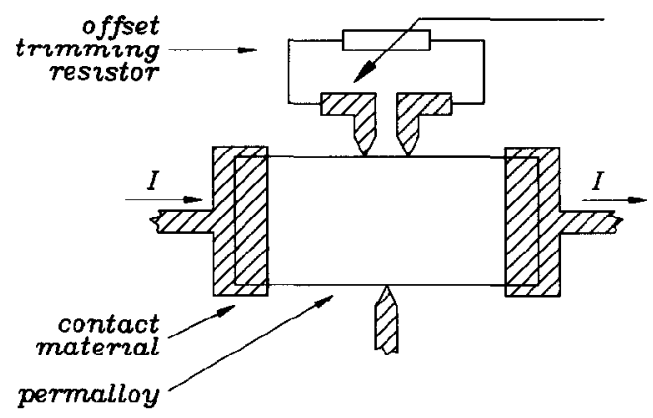

F1g 3 Offset correction circuit

the contacts The changes in offset after annealing at $125^{\circ} \mathrm{C}$ for longer times are generally lower than $075 \%$ We can conclude that offset attributes less than $175 \%$ to the signal under normal conditions in the temperature range $20^{\circ} \mathrm{C}$ to $120^{\circ} \mathrm{C}$, leading to angle detector measurement errors of $05^{\circ}$ maximum

It is concluded that the offset stability is the limiting property with regard to the accuracy of the angle detector Further research will focus on this problem

\section{Conclusions}

With the techniques described above, angle detectors with an accuracy of $05^{\circ}$ over a temperature range of 20 to $120^{\circ} \mathrm{C}$ can be produced There is no indication that the temperature range cannot be extended to lower temperatures The accuracy is mainly limited by temperature-induced changes in the offset of the devices If the technology can be adapted to provide more stable contacts, an accuracy of 02 to $01^{\circ}$ should be attainable

\section{Acknowledgement}

This work was supported by the Netherlands Technology Foundation (STW)

\section{References}

$1 \mathrm{~K} J \mathrm{M}$ Eujkel, A thu-film magnetoresustıve angle detector, $P h D$ Thests, University of Twente, Enschede, The Netherlands, 1988

2 C J M Eijkel and J H J Fluitman, Contactless angle transducer, US Patent Applic 06913760 (1986)

$3 \mathrm{~K} J \mathrm{M}$ Eykel and J H J Flutman, Optumization of the response of magnetoresistive elements, IEEE Trans Magn, (1989) in press

4 R F Soohoo, Magnetic Thin Fulms, Harper and Row, New York, 1965

$5 \mathrm{~J} \mathrm{~W}$ Wreberdink and $\mathrm{K} J \mathrm{M}$ Eijkel, Permalloy multılayers to reduce the effects of uniaxial anisotropy, $I E E E$ Trans Magn, (1989) in press

6 M Takayasu, S Uchyama, $\mathrm{K}$ Takahashı and T Fuju, Isothermal annealing behavior of zero-magnetostnctive permalloy films, IEEE Trans Magn MAG-10 (1974) 552555

$7 \mathrm{M}$ Goto, $\mathrm{H}$ Tange, T Kamimon and $\mathbf{H}$ Kubo, Dead anisotropy layer of 80-permalloy films evaporated in magnetic field, 12th Int Colloq Magnetic Fulms and Surfaces (ICMFS), France, 1988, ThP-15

8 W Metzdorf, Die Beeinflussbarkeit der uniaxualen magnetischen Anisotropie aufgedampfter Permalloyschichten durch Tempern im Magnetfeld, $Z$ Angew Phys, 18 (1964) $534-540$

9 T S Crowther, Angular and magnitude dispersion of the anisotropy in magnetic films, $J$ Appl Phys, 34 (1963) 580-586

$10 \mathrm{~K} \mathrm{~J} \mathrm{Eijkel,} \mathrm{Measurement} \mathrm{of} \mathrm{the} \mathrm{anisotropy} \mathrm{in} \mathrm{permalloy,}$ IEEE Trans Magn, MAG-24 (1988) 1957-1959

11 A D Butherus and S Nakahara, Annealing kınetics of thin permalloy films, IEEE Trans Magn, MAG-21 (1985) $1301-1305$

12 M Kitada and H Yamamoto, Reaction between permalloy and several thin metal films, Thin Solvd Films, 122 (1984) 173-182 